\title{
Doubly Periodic Riemann Boundary Value Problem of Non-Normal Type for Analytic Functions on Two Parallel Curves
}

\author{
Lixia Cao, Huijun Zheng \\ School of Mathematics and Statistics, Northeast Petroleum University, Daqing, China \\ Email: caolixia98237@163.com
}

Received 8 January 2015; accepted 22 January 2015; published 23 January 2015

Copyright (C) 2015 by authors and Scientific Research Publishing Inc.

This work is licensed under the Creative Commons Attribution International License (CC BY).

http://creativecommons.org/licenses/by/4.0/

(c) (i) Open Access

\begin{abstract}
In this paper, we present and study a kind of Riemann boundary value problem of non-normal type for analytic functions on two parallel curves. Making use of the method of complex functions, we give the method for solving this kind of doubly periodic Riemann boundary value problem of non-normal type and obtain the explicit expressions of solutions and the solvable conditions for it.
\end{abstract}

\section{Keywords}

Doubly Periodic, Holder Continuous Functions, Riemann Boundary Problem, Non-Normal Type

\section{Introduction}

Classical Riemann boundary value problems (RBVPs), doubly periodic or quasi-periodic RBVPs and Dirichlet Problems for analytic functions or for polyanalytic functions, on closed curves or on open arcs, have been widely investigated in papers [1]-[8]. The main approach is to use the decomposition of polyanalytic functions and their generalization to transform the given boundary value problems to their corresponding boundary value problems for analytic functions, and the fundamental and important tool for which is the Plemelj formula. Professor L. Xing proposed the Periodic Riemann Boundary Value Inverse Problems in paper [9], and then various inverse RBVPs for generalized analytic functions or bianalytic functions have been investigated in papers [10]-[13].

In present paper, we present a kind of doubly periodic RBVP of non-normal type for analytic functions on two parallel curves. On the basis of the results for normal type in paper [14], we give the method for solving this kind of doubly periodic RBVP of non-normal type and obtain the explicit expressions of solutions and the solvable conditions for it. 


\section{Doubly Periodic RBVP of Non-Normal Type on Two Parallel Curves}

Suppose that $\omega_{1}, \omega_{2}$ are complex constants with $\operatorname{Im}\left(\omega_{1} / \omega_{2}\right) \neq 0$, and $\mathbf{P}$ denotes the fundamental period parallelogram with vertices $\pm \omega_{1} \pm \omega_{2}$. The function

$$
\zeta(z)=1 / z+\sum_{m, n}^{\prime}\left[1 /\left(z-\Omega_{m n}\right)+1 / \Omega_{m n}+z / \Omega_{m n}^{2}\right]
$$

is called the Weierstrass $\zeta$-function, where $\Omega_{m n}=2 m \omega_{1}+2 n \omega_{2}$, and $\sum_{m, n}^{\prime}$ denotes the sum for all $m, n=0, \pm 1, \pm 2, \cdots$, except for $m=n=0$.

Let $L_{0}=\sum_{j=1}^{2} L_{0 j}$ be the set of two parallel curves, lying entirely in the fundamental period parallelogram $\mathbf{P}$, not passing the origin $O$, with endpoints being periodic congruent and having the same tangent lines at the periodic congruent points. Let $D_{1}, D_{2}, D_{3}$ denote the domains entirely in the fundamental period parallelogram $\mathbf{P}$, cut by $L_{01}$ and $L_{02}$, respectively. Without loss of generality, we suppose that $O \in D_{2}$ see Figure 1 . Let $L_{01}^{*}$, $L_{02}^{*}$ be the curves periodically extended for $L_{01}$ and $L_{02}$ with period $2 \omega_{1}$, respectively. And $L_{n j}^{*}$ $(j=1,2 ; n=0, \pm 1, \cdots)$ be the curves periodically extended for $L_{0 j}^{*}$ with $2 n \omega_{1}$.

We aim to is to find sectionally holomorphic, doubly periodic functions $F(z)$ and $\Omega(z)$, satisfying the following boundary conditions

$$
\begin{cases}F^{+}(\tau)=\frac{\Pi_{11}(\tau)}{\Pi_{12}(\tau)} D_{1}(\tau) \Omega^{-}(\tau)+g_{1}(\tau), & \tau \in L_{01}, \\ \Omega^{+}(\tau)=\frac{\Pi_{21}(\tau)}{\Pi_{22}(\tau)} D_{2}(\tau) F^{-}(\tau)+g_{2}(\tau), & \tau \in L_{02},\end{cases}
$$

where $D_{j}(\tau), g_{j}(\tau) \in H$ with $D_{j}(\tau) \neq 0(j=1,2)$, and $D_{j}(\tau), g_{j}(\tau)$ are doubly periodic with $2 \omega_{1}$, $2 \omega_{2} \cdot F^{ \pm}(\tau)$ are the boundary values of the function $F(z)$, which is analytic in $D_{1}$ and $D_{3}$, belonging to the class $h\left(a_{j}\right)$ on $L_{0 j}$, satisfying the boundary conditions (1), and $\Omega^{ \pm}(\tau)$ are the boundary values of the function $\Omega(z)$, which is analytic in $D_{2}$, belonging to the class $h\left(a_{j}\right)$ on $L_{0 j}$, satisfying the boundary conditions (1). While

$$
\begin{array}{cc}
\prod_{11}(\tau)=\prod_{s=1}^{h} \mu\left(\tau-c_{s}\right)^{\lambda_{s}}, & \Pi_{12}(\tau)=\prod_{l=1}^{p} \mu\left(\tau-d_{l}\right)^{u_{l}}, \\
\Pi_{21}(\tau)=\prod_{r=1}^{q} \mu\left(\tau-e_{r}\right)^{\delta_{r}}, & \Pi_{22}(\tau)=\prod_{v=1}^{w} \mu\left(\tau-t_{v}\right)^{n_{v}} . \\
\lambda=\sum_{s=1}^{h} \lambda_{s}, \quad u=\sum_{l=1}^{p} u_{l}, \quad \delta=\sum_{r=1}^{q} \delta_{r}, \quad n=\sum_{v=1}^{w} n_{v},
\end{array}
$$

where $\mu(\tau)=\frac{\sigma(\tau) \sigma\left(\tau-\omega_{1}-\omega_{2}\right)}{\sigma\left(\tau-\omega_{1}\right) \sigma\left(\tau-\omega_{2}\right)}$ is doubly periodic, where

$$
\sigma(\tau)=z \coprod_{k, t}^{\prime}\left(1-\frac{z}{\Omega_{k t}}\right) \exp \left\{\frac{z}{\Omega_{k t}}+\frac{z^{2}}{2 \Omega_{k t}^{2}}\right\}
$$

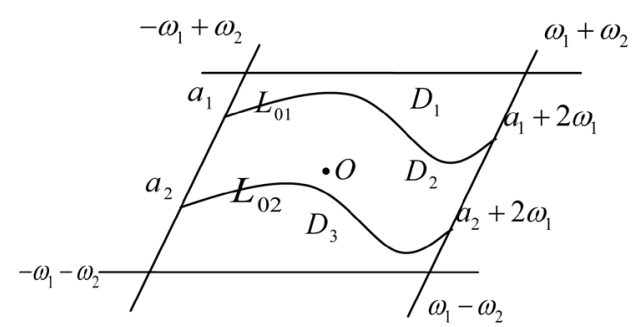

Figure 1. parallel curves in the fundamental period parallelogram $\mathbf{P}$. 
With $k, t$ and $\lambda_{s}, u_{l}, \delta_{r}, n_{v}$ being integers. Without loss of generality, we suppose that $c_{s}, d_{l} \in L_{01}$ with $c_{s}$, $d_{l} \neq a_{1}, \quad a_{1}+2 \omega_{1}(s=1, \cdots, h ; l=1, \cdots, p)$ as well as $e_{r}, f_{v} \in L_{02}$ and

$$
e_{r}, f_{v} \neq a_{1}, \quad a_{2}+2 \omega_{1} \quad(r=1, \cdots, q ; v=1, \cdots, w) .
$$

Since $a_{j}$ plays the same roles as other points on $L_{0 j}(j=1,2)$, it is natural to require that the unknown functions are bounded at $z=a_{j}$, that is, the unknown functions $F(z)$ and $\Omega(z)$ are both bounded on $L_{01}^{*}$ and $L_{02}^{*}$. And if we allow the solution $\Omega(z)$ has poles of order $m$ at $z=0$, it is actually to solve problem (1) in $D R_{m}$.

\section{Preliminary Notes}

Since $D_{j}(\tau) \in H$ with $D_{j}(\tau) \neq 0(j=1,2)$, by taking logarithm of $\log D_{j}(\tau)$ for some branch on $L_{0 j}$, we may obtain a continuous single-valued function such as

$$
\begin{aligned}
& -\frac{1}{2 \pi i} \log D_{j}\left(a_{j}\right)=\alpha_{a_{j}}+i \beta_{a_{j}}, \quad j=1,2, \\
& \frac{1}{2 \pi i} \log D_{j}\left(a_{j}+2 \omega_{j}\right)=-\alpha_{a_{j}}-i \beta_{a_{j}}, \quad j=1,2 .
\end{aligned}
$$

with $0 \leq \alpha_{a_{j}}<1$. Now we call the integer $\kappa=\kappa_{1}+\kappa_{2}$ the index of problem (1), where $\kappa_{j}$ is the integer satisfying

$$
0 \leq-\alpha_{a_{j}}-\kappa_{j}<1, \quad j=1,2 .
$$

Since $\kappa_{j}$ can only be 0 and -1 , the index $\kappa$ can only take $0,-1,-2$.

Set

$$
\begin{gathered}
D_{*}=D_{1^{*}}+D_{2 *}=\frac{1}{2 \pi i} \int_{L_{01}} \log D_{1}(\tau)+\frac{1}{2 \pi i} \int_{L_{02}} \log D_{2}(\tau) \mathrm{d} \tau \\
\gamma_{j}(z)=\frac{1}{2 \pi i} \int_{L_{0 j}} \log D_{j}(\tau) \xi(\tau-z) \mathrm{d} \tau, \quad z \notin L_{0 j}, \quad j=1,2
\end{gathered}
$$

We can easily see that $1 / \mathrm{e}^{\gamma_{j}(z)}$ will have singularities at most less than one order near the endpoints $a_{j}$ and $a_{j}+2 \omega_{1} \quad(j=1,2)$. Let

then we have

$$
\mathrm{e}^{\gamma(z)}=\mathrm{e}^{\gamma_{1}(z)} \mathrm{e}^{\gamma_{2}(z)}
$$

$$
\mathrm{e}^{\gamma\left(z+2 \omega_{j}\right)}=\mathrm{e}^{-2 \eta_{j} D_{*}} \mathrm{e}^{\gamma(z)}, \quad j=1,2,
$$

where $\eta_{j}=\zeta\left(\omega_{j}\right)(j=1,2)$ and $2 \omega_{2} \eta_{1}-2 \omega_{1} \eta_{2}=\pi i$. Thus $\mathrm{e}^{\gamma(z)}$ is not doubly periodic generally. In fact, $\mathrm{e}^{\gamma(z)}$ is doubly periodic if and only if

$$
\eta_{j} D_{*}=k_{j} \pi i, k_{j} \text { is positive integer for } j=1,2 .
$$

Lemma 1. Formula (5) is valid if and only if

$$
\eta_{1} / \eta_{2}=k_{1} / k_{2}, \quad D_{*}=2 k_{1} \omega_{2}-2 k_{2} \omega_{1} .
$$

And if both $D_{*}=2 l_{1} \omega_{1}+2 l_{2} \omega_{2}$ and $\eta_{j} D_{*}=k_{j} \pi i$ are true, then we have $l_{1}=-k_{2}$ and $l_{2}=k_{1}$, where $l_{j}$, $k_{j}$ are all integers.

\section{Solution for Problem (1)}

Problem (1) can be transferred as

$$
\begin{cases}\frac{\Pi_{12}(\tau) \Pi_{22}(\tau)}{\mathrm{e}_{1}^{\gamma_{1}^{+}(\tau)} \mathrm{e}^{\gamma_{2}^{-}(\tau)}} F^{+}(\tau)=\frac{\Pi_{11}(\tau) \Pi_{22}(\tau)}{\mathrm{e}^{\gamma_{1}^{-}(\tau)} \mathrm{e}^{\gamma_{2}^{-}(\tau)}} \Omega^{-}(\tau)+\frac{\Pi_{12}(\tau) \Pi_{22}(\tau)}{\mathrm{e}^{\gamma_{1}^{+}(\tau)} \mathrm{e}^{\gamma_{2}(\tau)}} g_{1}(\tau), & \tau \in L_{01}, \\ \frac{\Pi_{11}(\tau) \Pi_{22}(\tau)}{\mathrm{e}^{\gamma_{2}^{+}(\tau)} \mathrm{e}^{\gamma_{1}^{-}(\tau)}} \Omega^{+}(\tau)=\frac{\Pi_{21}(\tau) \Pi_{11}(\tau)}{\mathrm{e}^{\gamma_{2}^{-}(\tau)} \mathrm{e}^{\gamma_{1}^{-}(\tau)}} F^{-}(\tau)+\frac{\Pi_{11}(\tau) \Pi_{22}(\tau)}{\mathrm{e}^{\gamma_{1}^{-}(\tau)} \mathrm{e}^{\gamma_{2}^{+}(\tau)}} g_{2}(\tau), & \tau \in L_{02} .\end{cases}
$$


Case 1. If formula (5) holds, that is, $\mathrm{e}^{\gamma(z)}$ is doubly periodic, then by Lemma 1 we have

$$
D_{*} \equiv 0\left(\bmod 2 \omega_{1}, 2 \omega_{2}\right) .
$$

The function $1 / \mathrm{e}^{\gamma_{j}(z)}$ always has singularities less than one order near the endpoints $a_{j}$ and $a_{j}+2 \omega_{1}$ $(j=1,2)$ whatever $\kappa=0,-1,-2$. And then both

$$
\frac{\Pi_{12}(\tau) \Pi_{22}(\tau)}{\mathrm{e}^{\gamma_{1}^{+}(\tau)} \mathrm{e}^{\gamma_{2}^{-}(\tau)}} g_{1}(\tau) \text { and } \frac{\Pi_{11}(\tau) \Pi_{22}(\tau)}{\mathrm{e}^{\gamma_{1}^{-}(\tau)} \mathrm{e}^{\gamma_{2}^{+}(\tau)}} g_{2}(\tau)
$$

must belong to class $H$ or class $H^{*}$ on $L_{01}$ and $L_{02}$, respectively.

Set

$$
\begin{aligned}
& \Psi_{1}(z)=\frac{1}{2 \pi i} \int_{L_{01}} \frac{\Pi_{12}(\tau) \Pi_{22}(\tau)}{\mathrm{e}^{\gamma_{1}^{+}(\tau)} \mathrm{e}^{\gamma_{2}^{-}(\tau)}} g_{1}(\tau)[\zeta(\tau-z)+\zeta(z)] \mathrm{d} \tau, \quad z \notin L_{01} \\
& \Psi_{2}(z)=\frac{1}{2 \pi i} \int_{L_{02}} \frac{\Pi_{22}(\tau) \Pi_{11}(\tau)}{\mathrm{e}^{\gamma_{1}^{-}(\tau)} \mathrm{e}^{\gamma_{2}^{+}(\tau)}} g_{2}(\tau)[\zeta(\tau-z)+\zeta(z)] \mathrm{d} \tau, \quad z \notin L_{02},
\end{aligned}
$$

then (6) can be rewritten as

$$
\begin{cases}\frac{\Pi_{12}(\tau) \Pi_{22}(\tau)}{\mathrm{e}^{\gamma_{1}^{+}}(\tau) \mathrm{e}^{\gamma_{2}^{-}}(\tau)} F^{+}(\tau)-\Psi_{1}^{+}-\Psi_{2}^{+}=\frac{\Pi_{11}(\tau) \Pi_{22}(\tau)}{\mathrm{e}^{\gamma_{1}^{-}(\tau)} \mathrm{e}^{\gamma_{2}^{-}(\tau)}} \Omega^{-}(\tau)-\Psi_{1}^{-}-\Psi_{2}^{+}, & \tau \in L_{01}, \\ \frac{\Pi_{11}(\tau) \Pi_{22}(\tau)}{\mathrm{e}^{\gamma_{2}^{+}(\tau)} \mathrm{e}^{\gamma_{1}^{-}(\tau)}} \Omega^{+}(\tau)-\Psi_{1}^{-}-\Psi_{2}^{+}=\frac{\Pi_{11}(\tau) \Pi_{21}(\tau)}{\mathrm{e}^{\gamma_{2}^{-}(\tau)} \mathrm{e}^{\gamma_{1}^{-}(\tau)}} F^{-}(\tau)-\Psi_{1}^{-}-\Psi_{2}^{-}, & \tau \in L_{02},\end{cases}
$$

where $\Psi_{1}^{+}$and $\Psi_{2}^{+}$(or $\Psi_{1}^{-}$and $\Psi_{2}^{-}$) denote the boundary values of the functions $\Psi_{1}(z)$ and $\Psi_{2}(z)$. By the definitions of $\Psi_{1}(z)$ and $\Psi_{2}(z)$, we see that

(i) $\Psi_{1}^{+}(z)$ has no zeros in domain $D_{1}$;

(ii) The part of $\Psi_{1}^{-}(z)$ which has zeros in domain $D_{1} \cup D_{3}$ is $\Pi_{22}(z)$;

(iii) The part of $\Psi_{2}^{+}(z)$ which has zeros in domain $D_{1} \cup D_{2}$ is $\Pi_{11}(z)$;

(iv) $\Phi_{2}^{-}(z)$ has no zeros in domain $D_{3}$.

Write

$$
\Phi_{1}(z)= \begin{cases}\frac{\Pi_{12}(z) \Pi_{22}(z)}{\mathrm{e}^{\gamma(z)}} F^{+}(z)-\Psi_{1}^{+}(z)-\Psi_{2}^{+}(z), & z \in D_{1}, \\ \frac{\Pi_{11}(z) \Pi_{22}(z)}{\mathrm{e}^{\gamma(z)}} \Omega(z)-\Psi_{1}^{-}(z)-\Psi_{2}^{+}(z), & z \in D_{2}, \\ \frac{\Pi_{11}(z) \Pi_{21}(z)}{\mathrm{e}^{\gamma(z)}} F^{-}(z)-\Psi_{1}^{-}(z)-\Psi_{2}^{-}(z), & z \in D_{3} .\end{cases}
$$

When we solve problem (1) in $D R_{m}$, the unknown function $\Phi_{1}(z)$ is $n$-order at $z=0$. And now we will meet three kinds of situations in solving problem (1) in $D R_{m}$, according to the value of $m-\lambda-n$.

$1^{\circ}$ When $m-\lambda-n>0$, problem (1) is solvable without any restrictive conditions and the general solution is given by

$$
\left\{\begin{array}{l}
F^{+}(z)=\left[c_{0}+c_{1} \zeta^{\prime}(z)+\cdots+c_{m-\lambda-n-1} \zeta^{(m-\lambda-n-1)}(z)+\Psi_{1}^{+}(z)+\Psi_{2}^{+}(z)\right] \frac{\mathrm{e}^{\gamma(z)}}{\Pi_{12}(z) \Pi_{22}(z)}, \quad z \in D_{1}, \\
\Omega(z)=\left[c_{0}+c_{1} \zeta^{\prime}(z)+\cdots+c_{m-\lambda-n-1} \zeta^{(m-\lambda-n-1)}(z)+\Psi_{1}^{-}(z)+\Psi_{2}^{+}(z)\right] \frac{\mathrm{e}^{\gamma(z)}}{\Pi_{11}(z) \Pi_{22}(z)}, \quad z \in D_{2}, \\
F^{-}(z)=\left[c_{0}+c_{1} \zeta^{\prime}(z)+\cdots+c_{m-\lambda-n-1} \zeta^{(m-\lambda-n-1)}(z)+\Psi_{1}^{-}(z)+\Psi_{2}^{-}(z)\right] \frac{\mathrm{e}^{\gamma(z)}}{\Pi_{11}(z) \Pi_{21}(z)}, \quad z \in D_{3},
\end{array}\right.
$$

where $c_{0}, c_{1}, \cdots, c_{m-\lambda-n-1}$ are arbitrary constants. 
$2^{\circ}$ When $m-\lambda-n=0$, problem (1) is solvable if and only if the restrictive conditions

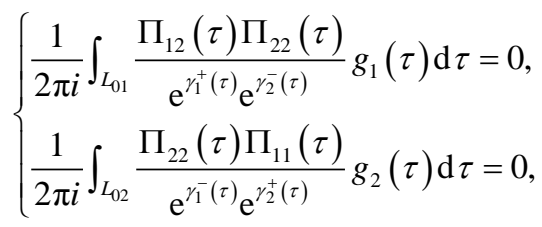

are satisfied, and now the solution is given by

$$
\begin{cases}F^{+}(z)=\frac{\mathrm{e}^{\gamma(z)}}{\Pi_{12}(\tau) \Pi_{22}(\tau)}\left[c+\Psi_{1}^{+}(z)+\Psi_{2}^{+}(z)\right], & z \in D_{1}, \\ \Omega(z)=\frac{\mathrm{e}^{\gamma(z)}}{\Pi_{11}(\tau) \Pi_{22}(\tau)}\left[c+\Psi_{1}^{-}(z)+\Psi_{2}^{+}(z)\right], & z \in D_{2}, \\ F^{-}(z)=\frac{\mathrm{e}^{\gamma(z)}}{\Pi_{11}(\tau) \Pi_{21}(\tau)}\left[c+\Psi_{1}^{-}(z)+\Psi_{2}^{-}(z)\right], & z \in D_{3},\end{cases}
$$

where $c$ is arbitrary constant.

$3^{\circ}$ When $m-\lambda-n<0, z=0$ is the zero point of order $m-\lambda-n$ of the function $\Phi_{1}(z)$, and due to this the solution for problem (1) has $-m$ order at the point $z=0$. Now the solution for problem (1) can still be given by (13), but the following two restrictive conditions are necessary:

$$
\begin{aligned}
& c=-\frac{1}{2 \pi i} \int_{L_{01}} \frac{g_{1}(\tau) \Pi_{12}(\tau) \Pi_{22}}{\mathrm{e}^{\gamma_{1}^{+}(\tau)} \mathrm{e}^{\gamma_{2}^{-}(\tau)}} \zeta(\tau) \mathrm{d} \tau-\frac{1}{2 \pi i} \int_{L_{02}} \frac{\Pi_{11}(\tau) \Pi_{22}(\tau)}{\mathrm{e}^{\gamma_{2}^{+}(\tau)} \mathrm{e}^{\gamma_{1}^{-}(\tau)}} g_{2}(\tau) \zeta(\tau) \mathrm{d} \tau=0, \\
& \left\{\begin{array}{l}
\frac{1}{2 \pi i} \int_{L_{01}} \frac{\Pi_{12}(\tau) \Pi_{22}(\tau)}{\mathrm{e}^{\gamma_{1}^{+}(\tau)} \mathrm{e}^{\gamma_{2}(\tau)}} g_{1}(\tau) \zeta^{(k)}(\tau) \mathrm{d} \tau=0, \\
\frac{1}{2 \pi i} \int_{L_{02}} \frac{\Pi_{11}(\tau) \Pi_{22}(\tau)}{\mathrm{e}^{\gamma_{2}^{+}(\tau)} \mathrm{e}^{\gamma_{1}^{-}(\tau)}} g_{2}(\tau) \zeta^{(k)}(\tau) \mathrm{d} \tau=0,
\end{array}\right.
\end{aligned}
$$

(when $m-\lambda-n=-1$, the condition (15) is unnecessary).

Case 2. If formula (5) fails to hold, then by Lemma 1 we see that $D_{*} \neq 0$. Let

$$
h_{*}(z)=\sigma(z) / \sigma\left(z-D_{*}\right),
$$

then the function $\mathrm{e}^{\gamma(z)} h_{*}(z)$ become doubly periodic, and function $1 /\left[\mathrm{e}^{\gamma(z)} h_{*}(z)\right]$ has singularities at most less than one order near the endpoints $a_{j}$ and $a_{j}+2 \omega_{1}(j=1,2)$. Thus now, we can transform (6) to

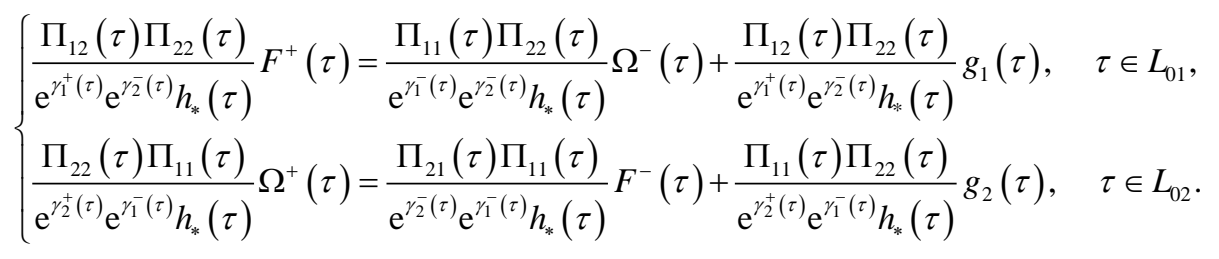

When $\kappa=0,-1,-2$, the two functions $\frac{\Pi_{12}(\tau) \Pi_{22}(\tau)}{\mathrm{e}^{\gamma_{1}^{+}(\tau)} \mathrm{e}^{\gamma_{2}^{2}(\tau)} h_{*}(\tau)} g_{1}(\tau), \frac{\Pi_{11}(\tau) \Pi_{22}(\tau)}{\mathrm{e}^{\gamma_{2}^{+}(\tau)} \mathrm{e}^{\gamma_{1}^{-}(\tau)} h_{*}(\tau)} g_{2}(\tau)$ belong to class $H$ or class $H^{*}$ on $L_{01}$ and $L_{02}$, respectively. Write

$$
\begin{aligned}
& \Psi_{1}(z)=\frac{1}{2 \pi i} \int_{L_{02}} \frac{\Pi_{11}(\tau) \Pi_{22}(\tau)}{\mathrm{e}^{\gamma_{2}^{+}(\tau)} \mathrm{e}^{\gamma_{1}^{-}(\tau)} h_{*}(z)} g_{2}(\tau)[\zeta(\tau-z)+\zeta(z)] \mathrm{d} \tau, \quad z \notin L_{01}, \\
& \Psi_{2}(z)=\frac{1}{2 \pi i} \int_{L_{02}} \frac{\Pi_{11}(\tau) \Pi_{22}(\tau)}{\mathrm{e}^{\gamma_{2}^{+}(\tau)} \mathrm{e}^{\gamma_{1}^{-}(\tau)} h_{*}(z)} g_{2}(\tau)[\zeta(\tau-z)+\zeta(z)] \mathrm{d} \tau, \quad z \notin L_{02} .
\end{aligned}
$$


By (17) and (18), we can rewrite (16) as

$$
\left\{\begin{array}{l}
\frac{\Pi_{12}(\tau) \Pi_{22}(\tau)}{\mathrm{e}^{\gamma_{1}^{+}(\tau)} \mathrm{e}^{\gamma_{2}^{-}(\tau)} h_{*}(\tau)} F^{+}(\tau)-\Psi_{1}^{+}(\tau)-\Psi_{2}^{+}(\tau)=\frac{\Pi_{11}(\tau) \Pi_{22}(\tau)}{\mathrm{e}^{\gamma_{1}^{-}(\tau)} \mathrm{e}^{\gamma_{2}^{-}(\tau)} h_{*}(\tau)} \Omega^{-}(\tau)-\Psi_{1}^{-}(\tau)-\Psi_{2}^{+}(\tau), \quad \tau \in L_{01}, \\
\frac{\Pi_{22}(\tau) \Pi_{11}(\tau)}{\mathrm{e}^{\gamma_{2}^{+}(\tau)} \mathrm{e}^{\gamma_{1}^{-}(\tau)} h_{*}(\tau)} \Omega^{+}(\tau)-\Psi_{1}^{-}(\tau)-\Psi_{2}^{+}(\tau)=\frac{\Pi_{21}(\tau) \Pi_{11}(\tau)}{\mathrm{e}^{\gamma_{2}^{-}(\tau)} \mathrm{e}^{\gamma_{1}^{-}(\tau)} h_{*}(\tau)} F^{-}(\tau)-\Psi_{1}^{-}(\tau)-\Psi_{2}^{-}(\tau), \quad \tau \in L_{02} .
\end{array}\right.
$$

Now we will meet two kinds of situations in solving problem (1) in $D R_{m}$.

(a) When $D_{*} \equiv 0 \bmod \left(2 \omega_{1}, 2 \omega_{2}\right)$, the function $h_{*}(z)$ is an entire function. And we can write it without counting nonzero constant as

$$
h_{*}(z)=\exp \left\{2\left(l_{1} \eta_{1}+l_{2} \eta_{2}\right)\right\}
$$

where $l_{1}, l_{2}$ are determined by the identity $D_{*}=2 l_{1} \omega_{1}+2 l_{2} \omega_{2}$.

$1^{\circ}$ When $m-\lambda-n>0$, problem (1) is solvable without any restrictive conditions and the general solution is given by

$$
\left\{\begin{array}{l}
F^{+}(z)=\left[c_{0}+c_{1} \zeta^{\prime}(z)+\cdots+c_{m-\lambda-n-1} \zeta^{(m-\lambda-n-1)}(z)+\Psi_{1}^{+}(z)+\Psi_{2}^{+}(z)\right] \frac{\mathrm{e}^{\gamma(z)} h_{*}(z)}{\Pi_{12}(z) \Pi_{22}(z)}, \quad z \in D_{1}, \\
\Omega(z)=\left[c_{0}+c_{1} \zeta^{\prime}(z)+\cdots+c_{m-\lambda-n-1} \zeta^{(m-\lambda-n-1)}(z)+\Psi_{1}^{-}(z)+\Psi_{2}^{+}(z)\right] \frac{\mathrm{e}^{\gamma(z)} h_{*}(z)}{\Pi_{11}(z) \Pi_{22}(z)}, \quad z \in D_{2}, \\
F^{-}(z)=\left[c_{0}+c_{1} \zeta^{\prime}(z)+\cdots+c_{m-\lambda-n-1} \zeta^{(m-\lambda-n-1)}(z)+\Psi_{1}^{-}(z)+\Psi_{2}^{-}(z)\right] \frac{\mathrm{e}^{\gamma(z)} h_{*}(z)}{\Pi_{11}(z) \Pi_{21}(z)}, \quad z \in D_{3},
\end{array}\right.
$$

where $c_{0}, c_{1}, \cdots, c_{m-\lambda-n-1}$ are arbitrary constants.

$2^{\circ}$ When $m-\lambda-n=0$, problem (1) is solvable if and only if the restrictive conditions

$$
\left\{\begin{array}{l}
\frac{1}{2 \pi i} \int_{L_{01}} \frac{\Pi_{12}(\tau) \Pi_{22}(\tau)}{\mathrm{e}^{\gamma_{1}^{+}(\tau)} \mathrm{e}^{\gamma_{2}^{-}(\tau)} h_{*}(\tau)} g_{1}(\tau) \mathrm{d} \tau=0, \\
\frac{1}{2 \pi i} \int_{L_{02}} \frac{\Pi_{11}(\tau) \Pi_{22}(\tau)}{\mathrm{e}^{\gamma_{2}^{+}(\tau)} \mathrm{e}^{\gamma_{1}^{-}(\tau)} h_{*}(\tau)} g_{2}(\tau) \mathrm{d} \tau=0,
\end{array}\right.
$$

are satisfied, and the general solution is given by

$$
\begin{cases}F^{+}(z)=\frac{\mathrm{e}^{\gamma(z)} h_{*}(z)}{\Pi_{12}(z) \Pi_{22}(z)}\left[c+\Psi_{1}^{+}(z)+\Psi_{2}^{+}(z)\right], & z \in D_{1} \\ \Omega(z)=\frac{\mathrm{e}^{\gamma(z)} h_{*}(z)}{\Pi_{11}(z) \Pi_{22}(z)}\left[c+\Psi_{1}^{-}(z)+\Psi_{2}^{+}(z)\right], & z \in D_{2}, \\ F^{-}(z)=\frac{\mathrm{e}^{\gamma(z)} h_{*}(z)}{\Pi_{11}(z) \Pi_{21}(z)}\left[c+\Psi_{1}^{-}(z)+\Psi_{2}^{-}(z)\right], & z \in D_{3}\end{cases}
$$

where $c$ is arbitrary constant.

$3^{\circ}$ When $m-\lambda-n<0$, problem (1) is solvable if and only if the restrictive conditions

$$
\left\{\begin{array}{l}
\frac{1}{2 \pi i} \int_{L_{02}} \frac{\Pi_{12}(\tau) \Pi_{22}(\tau) g_{1}(\tau)}{\mathrm{e}^{\gamma_{1}^{+}(\tau)} \mathrm{e}^{\gamma_{2}^{-}(\tau)} h_{*}(\tau)} \zeta^{(k)}(\tau) \mathrm{d} \tau=0, \\
\frac{1}{2 \pi i} \int_{L_{02}} \frac{\Pi_{11}(\tau) \Pi_{22}(\tau) g_{2}(\tau)}{\mathrm{e}^{\gamma_{2}^{+}(\tau)} \mathrm{e}^{\gamma_{1}^{-}(\tau)} h_{*}(\tau)} \zeta^{(k)}(\tau) \mathrm{d} \tau=0,
\end{array} \quad k=1,2, \cdots,-(m-\lambda-n)-1 .\right.
$$

are satisfied, and the general solution can still be given by (22) but with 


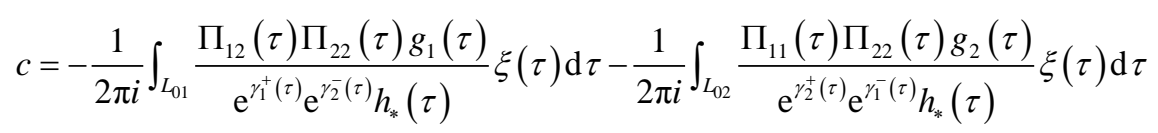

(b) When $D_{*} \equiv 0\left(\bmod 2 \omega_{1}, 2 \omega_{2}\right)$ fails to hold, the function $\frac{1}{\mathrm{e}^{\gamma(z)} h_{*}(z)}$ has singularity of one order at $z=0$, and has singularities at most less than one order near the endpoints $a_{j}$ and $a_{j}+2 \omega_{1}(j=1,2)$, has a zero of order one at $z=D_{*}$.

$1^{\circ}$ When $m-\lambda-n+1 \geq 0$, problem (1) is solvable and the general solution is given by

$$
\begin{cases}F^{+}(z)=\frac{\mathrm{e}^{\gamma(z)} h_{*}(z)}{\Pi_{12}(z) \Pi_{22}(z)}\left[c_{0}+c_{1} \zeta^{\prime}(z)+\cdots+c_{m-\lambda-n} \zeta^{(m-\lambda-n)}(z)+\Psi_{1}^{+}(z)+\Psi_{2}^{+}(z)\right], & z \in D_{1}, \\ \Omega(z)=\frac{\mathrm{e}^{\gamma(z)} h_{*}(z)}{\Pi_{11}(z) \Pi_{22}(z)}\left[c_{0}+c_{1} \zeta^{\prime}(z)+\cdots+c_{m-\lambda-n} \zeta^{(m-\lambda-n)}(z)+\Psi_{1}^{-}(z)+\Psi_{2}^{+}(z)\right], & z \in D_{2}, \\ F_{1}^{-}(z)=\frac{\mathrm{e}^{\gamma(z)} h_{*}(z)}{\Pi_{11}(z) \Pi_{21}(z)}\left[c_{0}+c_{1} \zeta^{\prime}(z)+\cdots+c_{m-\lambda-n} \zeta^{(m-\lambda-n)}(z)+\Psi_{1}^{-}(z)+\Psi_{2}^{-}(z)\right], & z \in D_{3},\end{cases}
$$

with the restrictive condition that

$$
c_{0}=-c_{1} \zeta^{\prime}\left(D_{*}\right)-\cdots-c_{m-\lambda-n} \zeta^{(m-\lambda-n)}\left(D_{*}\right)-\Psi_{1}\left(D_{*}\right)-\Psi_{2}\left(D_{*}\right)=0,
$$

which is to ensure that the solution be finite at $z=D_{*}$, where $c_{1}, c_{2}, \cdots, c_{m-\lambda-n}$ are arbitrary constants.

$2^{\circ}$ When $m-\lambda-n+1=-1$, problem (2.1) is solvable if and only if the restrictive conditions

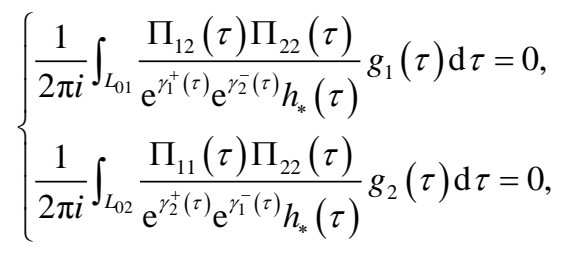

are satisfied, and now the solution is given by

$$
\begin{cases}F^{+}(z)=\frac{\mathrm{e}^{\gamma(z)} h_{*}(z)}{\Pi_{12}(z) \Pi_{22}(z)}\left[\Psi_{1}^{+}(z)+\Psi_{2}^{+}(z)-\Psi_{1}\left(D_{*}\right)-\Psi_{2}\left(D_{*}\right)\right], & z \in D_{1}, \\ \Omega(z)=\frac{\mathrm{e}^{\gamma(z)} h_{*}(z)}{\Pi_{11}(z) \Pi_{22}(z)}\left[\Psi_{1}^{-}(z)+\Psi_{2}^{+}(z)-\Psi_{1}\left(D_{*}\right)-\Psi_{2}\left(D_{*}\right)\right], & z \in D_{2}, \\ F^{-}(z)=\frac{\mathrm{e}^{\gamma(z)} h_{*}(z)}{\Pi_{11}(z) \Pi_{21}(z)}\left[\Psi_{1}^{-}(z)+\Psi_{2}^{-}(z)-\Psi_{1}\left(D_{*}\right)-\Psi_{2}\left(D_{*}\right)\right], & z \in D_{3},\end{cases}
$$

which is finite at $z=D_{*}$ owing to its structure.

$3^{\circ}$ When $m-\lambda-n+1<-1$, if and only if both conditions (26) and the following conditions

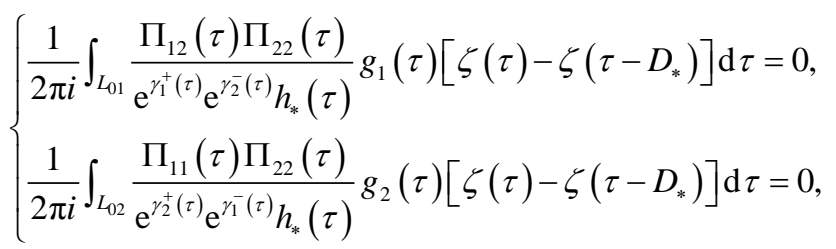

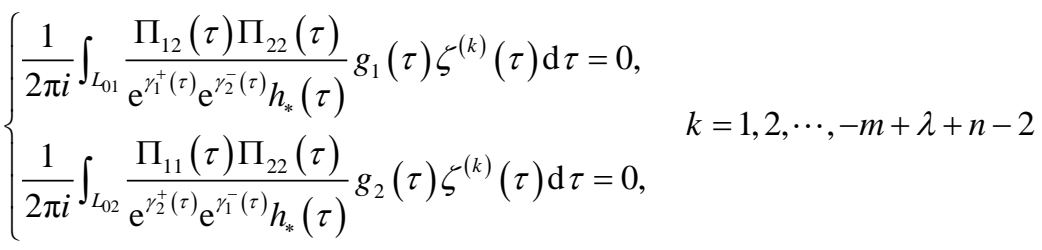


(when $m-\lambda-n+1=-2$, (29) is unnecessary) are satisfied, problem (1) is solvable and the solution is given by

$$
\begin{cases}F^{+}(z)=\frac{\mathrm{e}^{\gamma(z)} h_{*}(z)}{\Pi_{12}(z) \Pi_{22}(z)}\left[\Psi_{1}^{+}(z)+\Psi_{2}^{+}(z)-\Psi_{1}(0)-\Psi_{2}(0)\right], & z \in D_{1}, \\ \Omega(z)=\frac{\mathrm{e}^{\gamma(z)} h_{*}(z)}{\Pi_{11}(z) \Pi_{22}(z)}\left[\Psi_{1}^{-}(z)+\Psi_{2}^{+}(z)-\Psi_{1}(0)-\Psi_{2}(0)\right], \quad z \in D_{2}, \\ F^{-}(z)=\frac{\mathrm{e}^{\gamma(z)} h_{*}(z)}{\Pi_{11}(z) \Pi_{21}(z)}\left[\Psi_{1}^{-}(z)+\Psi_{2}^{-}(z)-\Psi_{1}(0)-\Psi_{2}(0)\right], & z \in D_{3} .\end{cases}
$$

\section{Funding}

The project of this thesis is supported by "Heilongjiang Province Education Department Natural Science Research Item", China (12541089).

\section{References}

[1] Balk, M.B. (1991) Polyanalytic Functions. Akademie Verlag, Berlin.

[2] Begehr, H. and Kumar, A. (2005) Boundary Value Problems for the Inhomogeneous Polyanalytic Equation I. Analysis: International Mathematical Journal of Analysis and its Application, 25, 55-71.

[3] Du, J.Y. and Wang, Y.F. (2003) On Boundary Value Problems of Polyanalytic Functions on the Real Axis. Complex Variables, 48, 527-542. http://dx.doi.org/10.1080/0278107031000103412

[4] Fatulaev, B.F. (2001) The Main Haseman Type Boundary Value Problem for Metaanalytic Function in the Case of Circular Domains. Mathematical Modelling and Analysis, 6, 68-76.

[5] Lu, J.K. (1993) Boundary Value Problems for Analytic Functions. World Scientific, Singapore.

[6] Mshimba, A.S. (2002) A Mixed Boundary Value Problem for Polyanalytic Function of Order $n$ in the Sobolev Space Wn, $p(D)$. Complex Variables, 47, 278-1077.

[7] Muskhelishvili, N.I. (1993) Singular Integral Equations. World Scientific, Singapore.

[8] Wanf, Y.F. and Du, J.Y. (2006) Hilbert Boundary Value Problems of Polyanalytic Functions on the Unit Circumference. Complex Variables and Elliptic Equations, 51, 923-943. http://dx.doi.org/10.1080/17476930600667692

[9] Xing, L. (1995) A Class of Periodic Riemann Boundary Value Inverse Problems. Proceedings of the Second Asian Mathematical Conference, Nakhon Ratchasima, October 1995, 397-400.

[10] Wang, M.H. (2006) Inverse Riemann Boundary Value Problems for Generalized Analytic Functions. Journal of Ningxia University of Natural Resources and Life Sciences Education, 27, 18-24.

[11] Wen, X.Q. and Li, M.Z. (2004) A Class of Inverse Riemann Boundary Value Problems for Generalized Holomorphic Functions. Journal of Mathematical, 24, 457-464.

[12] Cao, L.X., Li, P.-R. and Sun, P. (2012) The Hilbert Boundary Value Problem With Parametric Unknown Function on Upper Half-Plane. Mathematics in Practice and Theory, 42, 189-194.

[13] Cao, L.X. (2013) Riemann Boundary Value Problem of Non-Normal Type on the Infinite Straight Line. Applied Mathematics, 4, 1126-1230.

[14] Cao, L.X., Li, X.W. and Lin, C.X. (2014) A Kind of Doubly Periodic Riemann Boundary Value Problem on Two Parallel Curves. Advances in Pure Mathematics, 4, 627-634. 
Scientific Research Publishing (SCIRP) is one of the largest Open Access journal publishers. It is currently publishing more than 200 open access, online, peer-reviewed journals covering a wide range of academic disciplines. SCIRP serves the worldwide academic communities and contributes to the progress and application of science with its publication.

Other selected journals from SCIRP are listed as below. Submit your manuscript to us via either submit@scirp.org or Online Submission Portal.
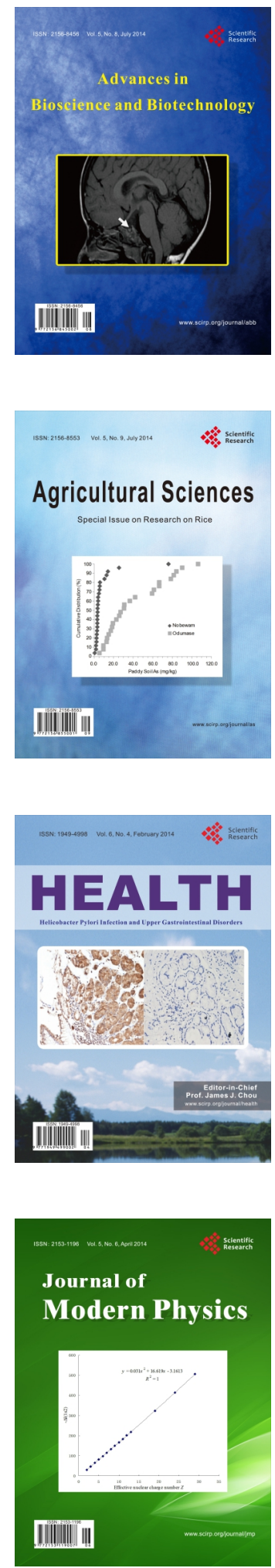
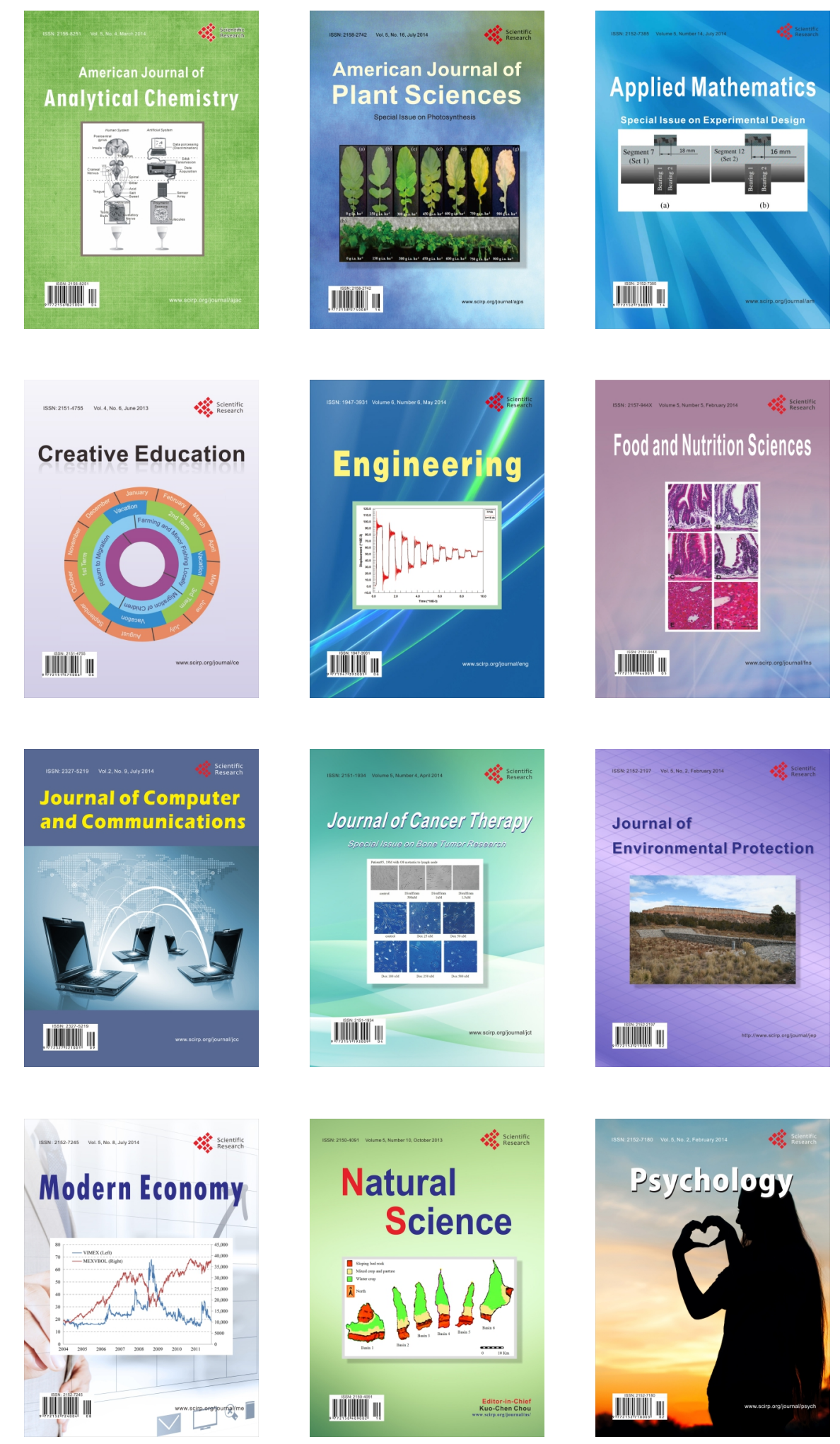